\title{
Generador de flujo con presión binivelada (BiPAP) a través de traqueostomía
}

\author{
SOLEDAD MONTES F. ${ }^{1}$, MIREYA MÉNDEZ R. ${ }^{2}$, PATRICIO BARAÑAO G. ${ }^{3}$, \\ PAMELA SALINAS F. ${ }^{4}$, FRANCISCO PRADO A. ${ }^{5}$ \\ 1. Hospital Josefina Martínez. \\ 2. Hospital Josefina Martínez, Departamento de Pediatría, Pontificia Universidad Católica de Chile. \\ 3. Kinesiólogo, Hospital Josefina Martínez. \\ 4. Enfermera, Hospital Josefina Martínez, Programa Asistencia Ventilatoria No Invasiva-MINSAL. \\ 5. Hospital Josefina Martínez, Sección Respiratorio, Departamento de Pediatría, Pontificia Universidad Católica de Chile, \\ Programa Asistencia Ventilatoria No Invasiva-MINSAL.
}

\begin{abstract}
Flow generator with double level pressure (BiPAP) through Tracheostomy

Background: The Chilean Program of Noninvasive Home Ventilation started using flow generating equipment with differential pressure at 2 levels (BiPAP) through tracheostomies for prolonged mechanical ventilation (PMV). Objective: Describe the experience of this ventilatory support, reporting selection criteria, procedure and technological requirements. Method: Descriptive-transversal study that includes 20 patients treated at Hospital Josefina Martínez, other pediatric hospitals and at home, for 12 months since June 2006. The clinical features, ventilation support, technical characteristics, follow-up and complications were reported. Results: The mean age was 3.5 years-old (range 3 months - 17 years). The duration of PMV ranged between 1 month to 5 years. Six patients $(30 \%)$ are at home and $14(70 \%)$ are hospitalized. In 14 patients $(70 \%)$, the need of PMV was due to neuromuscular diseases. There was no mortality related to the use of Bipap through tracheostomy; only 4 patients had minor complications. Conclusions: This report suggests that the use of BiPAP through tracheostomy in patients with selection criteria is an applicable PVM method. However, comparative systematic trials are necessary to define costs, benefits and risks of this type of ventilation.

(Key words: prolonged mechanical ventilation, Bipap, tracheostomy).

Rev Chil Pediatr 2008; 79 (5): 471-480

\section{RESUMEN}

Introducción: El Programa Chileno de Ventilación No Invasiva en domicilio (AVNI) extendió su cobertura utilizando generadores de flujo con presión bi-nivelada (BiPAP) en niños con ventilación mecánica prolongada (VMP) y traqueostomía (TQT). Objetivo: Reportar la experiencia de esta estrategia describiendo criterios de selección, modalidades de uso y tecnologías complementarias. Pacientes y Métodos: Estudio descriptivo, transversal y prospectivo durante un año desde Junio 2006, en 20 pacientes maneja-
\end{abstract}

Trabajo recibido el 06 de mayo de 2008, devuelto para corregir el 08 de septiembre de 2008, segunda versión el 10 de septiembre de 2008, aceptado para publicación el 10 de septiembre de 2008 .

Correspondencia a:

Francisco Prado A.

E-mail: panchoprado2004@yahoo.com 
dos en el Hospital Josefina Martínez, otros centros de la red asistencial del Ministerio de Salud y en domicilio. Se registraron las características clínicas, modos ventilatorios, evolución y complicaciones. Resultados: La mediana de edad fue de 3,5 años (rango 3 meses a 17 años). La duración de la VMP fue 1m a 5a, 6 pacientes $(30 \%)$ se encuentran en domicilio y $14(70 \%)$ hospitalizados. La principal causa para VMP fue enfermedad neuromuscular $(14,70 \%)$. No hubo mortalidad y 4 pacientes tuvieron complicaciones menores. Conclusión: El BiPAP a través de TQT, usado con criterios estrictos de selección, es un método de VMP que puede ser factible. Se requieren estudios comparativos para definir costos, beneficios y riesgos de estos equipos comparándolos con ventiladores licenciados para soporte vital.

(Palabras clave: Ventilación mecánica prolongada, BiPAP a través de traqueostomía).

Rev Chil Pediatr 2008; 79 (5): 471-480

\section{Introducción}

Con los avances diagnósticos y terapéuticos, la sobrevida de los pacientes con trastornos neuromusculares y con daño pulmonar crónico ha mejorado considerablemente ${ }^{1-5}$. La indicación temprana de ventilación no invasiva (VNI) al momento de comprobar hipoventilación nocturna ha demostrado mejorar la calidad de vida como también parámetros clínicos y funcionales, principalmente en pacientes con enfermedad neuromuscular6,7. Otro aspecto importante se relaciona con la reducción en los costos del manejo de estos pacientes al disminuir la frecuencia de hospitalizaciones generalmente por exacerbaciones respiratorias secundarias a neumonías y atelectasias ${ }^{6,8}$.

Sin embargo, distintas publicaciones, guías y normas en el manejo domiciliario de estos pacientes, han señalado que la ventilación mecánica invasiva esta indicada cuando la reserva ventilatoria impide su manejo con intervalos libres de soporte de presión positiva en la vía aérea, sobre todo en aquellos con mal manejo de las secreciones, tos débil y ausencia de reflejos deglutorios, condiciones que obligan a realizar una traqueostomía para ventilar en forma prolongada ${ }^{8-10}$.

En Chile, desde el año 2006 se cuenta con un programa gubernamental destinado a cubrir las necesidades de VNI domiciliaria nocturna en pacientes menores de 20 años ${ }^{11}$. Al momento de esta publicación 137 pacientes han ingresado, 95 (70\%) tienen una enfermedad neuromuscular y la indicación para VNI esta asociada a síndrome de hipoventilación nocturna o síndrome de apnea obstructiva del sueño (SAOS). No obstante, ha sido necesario exten- der la cobertura de las prestaciones a pacientes con ventilación mecánica prolongada (VMP), quienes requieren de una traqueostomía ${ }^{12}$.

Con el fin de planificar las estrategias apropiadas que permitan abordar este desafío y llegar a construir un Programa de ventilación mecánica invasiva (VMI), complementario al de VNI domiciliaria, es necesario distinguir con claridad dos grupos de pacientes. El primero constituido por aquellos con necesidad de traqueostomía y asistencia ventilatoria con equipos tradicionales ciclados las 24 horas del día, sin intervalo libre o ventanas de desconexión y con ausencia de los mecanismos de protección glótica que generalmente ha obligado a alimentar por gastrostomía. Estos pacientes suelen tener severamente comprometido su comando ventilatorio y la capacidad de generar trabajo respiratorio propio. El segundo grupo, esta constituido por pacientes con debilidad de la bomba, esfuerzo ventilatorio deficiente o con síndrome de apnea obstructiva del sueño, todos eventos patológicos que se potencian al dormir, pero pueden ser manejados apropiadamente con generadores de flujo y presión en dos niveles (BiPAP) con interfases constituidas por mascarillas nasales o nasobucales, evitando el requerimiento de una vía aérea artificial, es decir tributarios de ventilación mecánica no invasiva. Sin embargo, es posible identificar un tercer grupo constituido por pacientes que requieren traqueostomía, que han estado ventilados en forma estable por largo tiempo por enfermedades pulmonares, de la vía aérea o compromiso de la caja torácica, en los cuales existe buena tolerancia a la presión de soporte ventilatorio (PSV) o es posible períodos de desconexión o ventanas sin soporte de presión (comando 
ventilatorio total o parcialmente conservado), incluso algunos de ellos pueden derivar desde la ventilación invasiva a la no invasiva.

En los pacientes de este grupo de complejidad intermedia y también en el primer grupo de ellos han existido experiencias aisladas y no sistemáticas de conexión de BiPAP a través de traqueostomía (TQT) ${ }^{12,13}$. No existe en la literatura médica evidencia de la posible eficacia y seguridad del uso de estos equipos para entregar ventilación mecánica prolongada a través de una TQT. El objetivo de esta publicación es describir la metodología, características clínicas y requerimientos tecnológicos para la entrega de VMP a través de TQT, establecidas en el Programa Nacional de Asistencia Ventilatoria No Invasiva en Chile.

\section{Material y Métodos}

Se realizó un corte transversal y seguimiento durante un año (Junio 2006-Julio 2007), del registro en las fichas clínicas de 20 pacientes en régimen de VMP con BiPAP a través de TQT manejados en los siguientes escenarios

\section{Tabla 1. Criterio de inclusión BiPAP a través de traqueostomía}

1. Niños en ventilación mecánica invasiva prolongada: a través de traqueostomía con ventiladores convencionales por más de 21 días y con más de 6 horas de requerimientos ventilatorios continuos al día

2. Condición clínica estable, sin cambios en sus parámetros ventilatorios o clínicos en los 30 días previos al ingreso a protocolo. (VMIS + PSV)

3. Requerimientos de $\mathrm{FiO}_{2}$ menor de $0,3^{*}$

4. Paciente con traqueostomía que se encuentren sin soporte ventilatorio, pero en las siguientes condiciones clínicas

a) Aumento del trabajo respiratorio definido por polipnea mayor a 2 DS para la edad

b) Hipoventilación definida por: hipercapnea $\left(\mathrm{PaCO}_{2}\right.$ mayor de $50 \mathrm{mmHg}$ ) o pobre auscultación del murmullo pulmonar en planos posterobasales-axilares

c) Posterior a establecer CPAP mayor o igual a $5 \mathrm{~cm} \mathrm{H} O$ presenten disminución de la polipnea, disminución de la hipercapnea y/o mejoría en la auscultación pulmonar

5. Consentimiento informado.

VMIS: ventilación mandatoria intermitente sincronizada, PSV: presión de soporte ventilatoria. clínicos: a) Hospital Josefina Martínez u otros centros pediátricos de la red asistencial del Ministerio de Salud de Chile; b) en domicilio bajo supervisión del Programa Nacional de Asistencia Ventilatoria No Invasiva (www.avni.cl) o que estuvieran en hospitalización domiciliaria pública o privada cumpliendo con los estándares de manejo y prestaciones definidos por el Programa de Asistencia Ventilatoria Domiciliaria del Servicio de Salud Metropolitano Sur Oriente $^{12} \mathrm{y}$ del actual protocolo de VMI ${ }^{14}$. Los criterios de inclusión y exclusión considerados para la ventilación mecánica invasiva prolongada administrada con BiPAP a través de TQT se presentan en las tablas 1 y 2 .

\section{Modalidad de ventilación con BiPAP a traqueostomía}

Se usó cánula de TQT sin balón (vinílica) cuyas dimensiones fueron las apropiadas para el diámetro de la vía aérea. En los pacientes sin obstrucción de la vía aérea supraostoma se usó válvula de fonación Shiley en los períodos de ventana o Passy-Muir en aquellos con dependencia ventilatoria completa.

Los equipos generadores de flujo con presión bi-nivelada utilizados fueron BiPAP modelo Harmony (Respironics, USA) en 17 pacientes, 2 pacientes con BiPAP Synchrony (Respironics, USA) y uno con BiPAP S/T-D30 (Respironics, USA). Para la conexión a la TQT se usó un corrugado corto (Omniflex) que permite la movilidad, disminuyendo el riesgo de desconexión o decanulación, un conector $15 / 22 \mathrm{~mm}$ (diámetro interno/diámetro externo) con una

Tabla 2. Criterios de exclusión BiPAP a través de traqueostomía

1. Niños con condición clínica o parámetros ventilatorios inestables, $\mathrm{FiO}_{2}$ mayor a 0,3*

2. Requerimientos de soporte ventilatorio menor a $6 \mathrm{hrs} /$ día

3. Padres o paciente mayor de 15 años que rehúsa la terapia

4. Mala tolerancia o fracaso definido como uno o todos los eventos que se detallan: desaturación, taquicardia o bradicardia, hipoventilación definida como pobre auscultación en planos posterobasales y axilares de los campos pulmonares o aumento de la $\mathrm{PaCO}_{2}$ en más de $4 \mathrm{mmHg}$ del basal o $\mathrm{pH}$ menor a 7,20

* Los equipos utilizados no disponen de mezclador aire-oxígeno, por lo que no tiene un perfil de seguridad adecuado para pacientes con requerimientos mayores. 
perforación de 2,5 $\mathrm{mm}$, para impedir la recirculación de $\mathrm{CO}_{2}$ (portal exhalatorio) y un adaptador para inhalador de dosis medida (IDM). El circuito para conexión fue un corrugado de 22 $\mathrm{mm}$ con superficie interna lisa (figura 1 ). Todos los pacientes recibieron humidificación activa con base termohumedificador Respironics modelo Remstar (Respironics, USA) o con base calefactora modelos HC-150 y MR-810 (Fisher \& Paykel, New Zealand) con cámara humidificadora de sobrepaso MR-290 (Fisher \& Paykel, New Zealand). Según necesidad para impedir la condensación de agua en el circuito se usó trampa de agua, cable endocalefactor (sólo en caso de base termohumedificadora MR-810) o funda de polietileno para cubrir el corrugado.

Salvo excepciones, en pacientes mayores de 10 años que no presentaron complicaciones (secreciones espesas, broncoconstricción $\mathrm{u}$ otros elementos clínicos vinculados a pobre

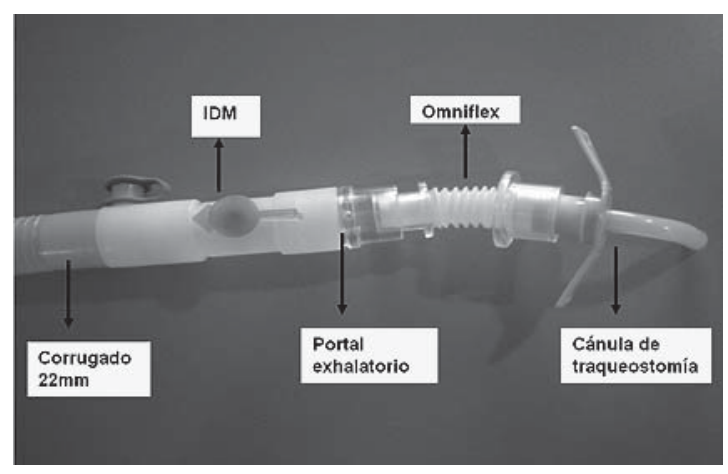

Figura 1. Insumos necesarios para entregar ventilación mecánica prolongada con BiPAP a través de traqueostomía.

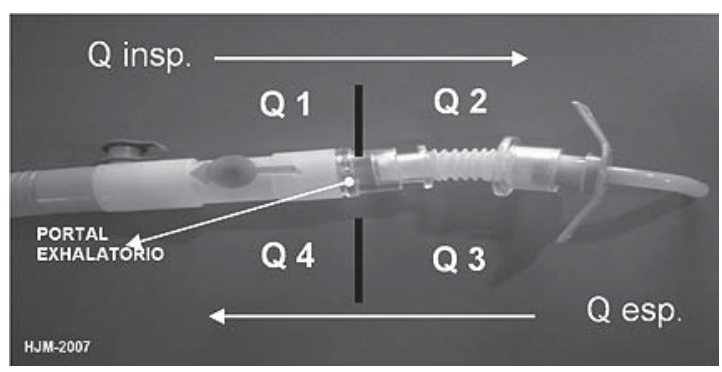

Figura 2. Diagrama de los sitios para la medición de los flujos en el circuito de conexión. Q1: Ventilometría inspiratoria pre-portal exhalatorio; Q2: Ventilometría inspiratoria posterior al portal exhalatorio; Q3: Ventilometría espiratoria pre-portal exhalatorio; Q4: Ventilometría espiratoria post-portal exhalatorio. termohumedificación de la vía aérea) se usó humidificación pasiva con dispositivos heat and moisture exchanger (HME). En aquellos pacientes sin intervalos libres de desconexión se usó una batería externa (Respironics, USA) con un máximo de autonomía de 8 horas según los parámetros programados. Las alarmas internas de los equipos para desconexión se fijaron en 15 segundos. En el caso de utilizar BiPAP Synchrony y en pacientes de 20 kilos o más de peso se utilizó la modalidad de asistencia de presión de soporte con volumen asegurado (AVAPS) que permite, ciclando por tiempo y en el rango de una presión positiva inspiratoria en la vía aérea (IPAP) máxima y mínima, mantener un volumen corriente (VT) deseado. En esta modalidad se fijaron alarmas de volumen minuto mínimo (VE) y VT según el peso y frecuencia respiratoria (FR). Como monitorización cardiorrespiratoria se utilizó un oxímetro de pulso con registro de $\mathrm{SPO}_{2}$ y frecuencia cardíaca (FC). Las alarmas se fijaron en $90 \%$, 60 y 180 latidos por minuto respectivamente.

\section{Registro}

Se registraron los siguientes datos demográficos, generales y de ventilación: género, edad, diagnóstico, modelo del BiPAP, modalidad y parámetros de ventilación: presiones utilizadas (IPAP y presión positiva espiratoria en la vía aérea: EPAP), frecuencia respiratoria de respaldo, uso de AVAPS y flujo de oxígeno. Se registró el tipo de apoyo ventilatorio previo a la conexión a BiPAP (ausente o presente, especificando cual). En los pacientes ingresados al Hospital Josefina Martínez se registró el flujo total $(1 / \mathrm{m})$ del sistema y el flujo del portal exhalatorio con un ventilómetro de Wright marca Ferraris. Se realizarán 4 ventilometrías simultáneas por paciente: 1) Ventilometría inspiratoria pre-portal exhalatorio (Q1); 2) Ventilometría inspiratoria posterior al portal exhalatorio (Q2); 3) Ventilometría espiratoria pre-portal exhalatorio (Q3) y 4) Ventilometría espiratoria post-portal exhalatorio (Q4). La diferencia entre ambas mediciones inspiratorias ( 1 y 2 ), permite calcular la reducción del flujo inspiratorio. A su vez, la diferencia entre los flujos espiratorios (3 y 4), corresponde al flujo por el portal exhalatorio. Este impide la re-inhalación del 
aire espirado y por lo tanto la recirculación de $\mathrm{CO}_{2}$ (figura 2). Dentro del seguimiento de estos pacientes se realizaron 3 evaluaciones clínicas: 1) Preconexión a BiPAP; 2) Postconexión temprana: desde la conexión hasta 30 días posterior a ella; 3) Postconexión tardía: determinación más cercana al momento de este reporte. En estas evaluaciones se registraron los siguientes parámetros clínicos y de monitorización no invasiva: FR programada y total (FR respal- do más FR gatillada por el paciente), auscultación pulmonar de las bases (se califico en buena o mala), $\mathrm{FC}, \mathrm{SPO}_{2}$, uso de musculatura accesoria (presente o ausente) y requerimiento de oxígeno (flujo de oxígeno en $1 / \mathrm{min}$ ). Se controlaron gases arteriales o venosos (GSA, GSV), en forma simultánea al registro descrito o según criterio médico dependiendo del estado clínico de cada paciente. Además, se evaluó el grado de autonomía registrando el número y

Tabla 3. Diagnóstico relacionado a ventilación mecánica prolongada y modalidad de ventilación con BiPAP a través de traqueostomía

\begin{tabular}{|c|c|c|c|c|c|c|c|c|c|}
\hline $\begin{array}{l}N^{\circ} \\
\text { pcte }\end{array}$ & Diagnóstico & $\begin{array}{c}\text { Ventilación } \\
\text { previa }\end{array}$ & $\begin{array}{l}\text { Modalidad } \\
\text { BiPAP }\end{array}$ & $\begin{array}{l}\text { Tiempo } \\
\text { de uso }\end{array}$ & $\begin{array}{c}\text { IPAP } \\
\left(\mathrm{cmH}_{2} \mathrm{O}\right)\end{array}$ & $\begin{array}{c}\text { EPAP } \\
\left(\mathrm{cmH}_{2} \mathrm{O}\right)\end{array}$ & $\underset{(\text { rpm) }}{\text { FR }}$ & $\begin{array}{c}\mathbf{O}_{2} \\
\text { (It } / \mathrm{min})\end{array}$ & $\begin{array}{l}\text { Uso válvula } \\
\text { fonación }\end{array}$ \\
\hline 1 & $\begin{array}{l}\text { Daño pulmonar crónico, } \\
\text { secuela ADV }\end{array}$ & CPAP & $\mathrm{S} / \mathrm{T}$ & Ventana & 12 & 8 & 12 & 0,5 & Shiley \\
\hline 2 & Atrofia espinal tipo I & SIMV & S/TAVAPS & Continuo & $12-18$ & 6 & 12 & & No \\
\hline 3 & $\begin{array}{l}\text { Sd Di George-daño } \\
\text { pulmonar crónico, } \\
\text { Fallot operado, } \\
\text { Traqueobroncomalacia } \\
\text { severa }\end{array}$ & SIMV & $\mathrm{S} / \mathrm{T}$ & Sueño & 18 & 8 & 12 & 0,75 & Shiley \\
\hline 4 & $\begin{array}{l}\text { Bronquiolitis obliterante, } \\
\text { post Steven Johnson }\end{array}$ & SIMV & S/T AVAPS & Continuo & $16-20$ & 8 & 12 & 2 & PM \\
\hline 5 & $\begin{array}{l}\text { DBP y quiste laringeo } \\
\text { subglótico }\end{array}$ & NADA & $\mathrm{S} / \mathrm{T}$ & Sueño & 15 & 5 & 22 & & No \\
\hline 6 & $\begin{array}{l}\text { Sd Hipotónico, granu- } \\
\text { loma supraostoma }\end{array}$ & CPAP & $\mathrm{S} / \mathrm{T}$ & Sueño & 8 & 4 & 4 & & Shiley \\
\hline 7 & Atrofia espinal Tipo I & SIMV & $\mathrm{S} / \mathrm{T}$ & Ventana & 16 & 8 & 12 & & PM \\
\hline 8 & Miopatía congénita & SIMV & $\mathrm{S} / \mathrm{T}$ & Continuo & 14 & 6 & 10 & & No \\
\hline 9 & Encefalitis/paraparesia & SIMV & $\mathrm{S} / \mathrm{T}$ & Continuo & 9 & 6 & 10 & & No \\
\hline 10 & Miopatía mitocondrial & SIMV & $\mathrm{S} / \mathrm{T}$ & Continuo & 12 & 5 & 10 & & No \\
\hline 11 & $\begin{array}{l}\text { Distrofia muscular } \\
\text { congénita }\end{array}$ & SIMV & $\mathrm{S} / \mathrm{T}$ & Continuo & 14 & 8 & 10 & & No \\
\hline 12 & $\begin{array}{l}\text { Distrofia muscular } \\
\text { congénita }\end{array}$ & SIMV & $\mathrm{s}$ & Continuo & 14 & 7 & & 0,5 & No \\
\hline 13 & Sd. Guillian Barré & SIMV & S & Continuo & 12 & 8 & & & No \\
\hline 14 & Distrofia muscular familiar & SIMV & $\mathrm{S} / \mathrm{T}$ & Continuo & 14 & 8 & 8 & & No \\
\hline 15 & $\begin{array}{l}\text { Distrofia muscular } \\
\text { progresiva }\end{array}$ & SIMV & $\mathrm{S} / \mathrm{T}$ & Ventana & 15 & 5 & 15 & & No \\
\hline 16 & $\begin{array}{l}\text { Malasia-parálisis } \\
\text { diafragmática derecha }\end{array}$ & SIMV & $\mathrm{S} / \mathrm{T}$ & Continuo & 10 & 5 & 8 & & No \\
\hline 17 & Miopatía miotubular & SIMV & $\mathrm{S} / \mathrm{T}$ & Ventana & 14 & 6 & 10 & & PM \\
\hline 18 & Sd. Escobar & SIMV & $\mathrm{S} / \mathrm{T}$ & Ventana & 12 & 8 & 10 & 0,3 & Shiley \\
\hline 19 & Bronquiomalasia severa & NADA & $S / T$ & Continuo & 14 & 7 & 15 & 0,5 & No \\
\hline 20 & Miopatía nemalínica & SIMV & S/TAVAPS & Continuo & $16-20$ & 8 & 16 & & Shiley \\
\hline
\end{tabular}

SIMV: ventilación mandatoria intermitente sincronizada; CPAP: presión positiva continua en la vía aérea; PM: válvula de fonación Passy-Muir; S/T: modalidad asistida/controlada; S: modalidad espontánea; DBP: displasia broncopulmonar; AVAPS: soporte de presión volumen promedio asegurado; ADV: adenovirus. 
Tabla 4. Medición de los flujos en el circuito de conexión en pacientes con ventilación mecánica prolongada con BiPAP a través de traqueostomía

\begin{tabular}{rcccccc}
\hline $\begin{array}{c}\text { Flujo (L/m) } \\
\mathbf{N}^{\mathbf{*}} \text { Pcte (*) }\end{array}$ & Q1 & Q2 & Q3 & Q4 & $\begin{array}{c}\text { Q1-Q2 } \\
\text { Reducción flujo } \\
\text { inspiratorio }\end{array}$ & $\begin{array}{c}\text { Q3-Q4 } \\
\text { Flujo portal } \\
\text { exhalatorio }\end{array}$ \\
\hline 3 & 17 & 7,5 & 2 & 0 & 9,5 & 2 \\
4 & 17 & 12,5 & 7 & 0,2 & 4,5 & 6,8 \\
5 & 7 & 4 & 4 & 0 & 3 & 4 \\
6 & 9,5 & 4 & 3,7 & 0 & 5,5 & 3,7 \\
7 & 11,4 & 6 & 5 & 0,2 & 5,4 & 4,8 \\
19 & 14,5 & 4,5 & 2 & 0 & 10 & 2 \\
20 & 29 & 18 & 12 & 0,5 & 11 & 11,5 \\
Mediana & 14,5 & 6 & 4 & 0 & 5,5 & 4 \\
\hline
\end{tabular}

(*) Referido a descripción de los pacientes tabla 3; Q1 Ventilometría inspiratoria pre-portal exhalatorio; Q2 Ventilometría inspiratoria posterior al portal exhalatorio; Q3 Ventilometría espiratoria pre-portal exhalatorio; Q4 Ventilometría espiratoria post-portal exhalatorio. La diferencia entre mediciones inspiratorias (Q1 y Q2), permite calcular la reducción del flujo inspiratorio. La diferencia entre los flujos espiratorios (Q3 y Q4), corresponde al flujo por el portal exhalatorio, leak exhalatorio o válvula exhalatoria.

Tabla 5. Registro de gases sanguíneos antes y después de la ventilación mecánica prolongada a través de traqueostomía con BiPAP

\begin{tabular}{|c|c|c|c|c|c|c|c|c|}
\hline $\mathbf{N}^{\circ}$ Pcte* & $\begin{array}{l}\text { Gases } \\
\text { Sanguíneos }\end{array}$ & Tiempos & pH & $\mathrm{PCO}_{2}$ & $\mathrm{PO}_{2}$ & $\mathrm{HCO}_{3}$ & $\mathrm{SpO}_{2}$ & $\mathbf{F R}$ \\
\hline 2 & Arterial & $\begin{array}{l}\text { Preconexión } \\
\text { Post conexión temprana } \\
\text { Post conexión tardía }\end{array}$ & $\begin{array}{l}7,48 \\
7,54 \\
7,48\end{array}$ & $\begin{array}{l}30 \\
23 \\
19\end{array}$ & $\begin{array}{r}90 \\
82 \\
101\end{array}$ & $\begin{array}{l}22,5 \\
20 \\
14,5\end{array}$ & $\begin{array}{l}98 \\
98 \\
98\end{array}$ & $\begin{array}{l}12 \\
12 \\
12\end{array}$ \\
\hline 3 & Venosa & $\begin{array}{l}\text { Preconexión } \\
\text { Post conexión temprana } \\
\text { Post conexión tardía }\end{array}$ & $\begin{array}{l}7,4 \\
7,37 \\
7,39\end{array}$ & $\begin{array}{l}31 \\
43 \\
39\end{array}$ & $\begin{array}{l}59 \\
38 \\
51\end{array}$ & $\begin{array}{l}23 \\
25 \\
25\end{array}$ & $\begin{array}{l}97 \\
94 \\
94\end{array}$ & $\begin{array}{l}12 \\
12 \\
12\end{array}$ \\
\hline 4 & Venosa & $\begin{array}{l}\text { Preconexión } \\
\text { Post conexión temprana } \\
\text { Post conexión tardía }\end{array}$ & $\begin{array}{l}7,29 \\
7,21 \\
7,33\end{array}$ & $\begin{array}{l}52 \\
65 \\
29\end{array}$ & $\begin{array}{r}123 \\
28 \\
112\end{array}$ & $\begin{array}{l}25 \\
25 \\
15\end{array}$ & $\begin{array}{l}97 \\
96 \\
96\end{array}$ & $\begin{array}{r}8 \\
12 \\
12\end{array}$ \\
\hline 5 & Venosa & $\begin{array}{l}\text { Preconexión } \\
\text { Post conexión temprana } \\
\text { Post conexión tardía }\end{array}$ & $\begin{array}{l}7,45 \\
7,39 \\
7,35\end{array}$ & $\begin{array}{l}47 \\
66 \\
33\end{array}$ & $\begin{array}{l}34 \\
38 \\
64\end{array}$ & $\begin{array}{l}33 \\
40 \\
18,5\end{array}$ & $\begin{array}{l}96 \\
98 \\
98\end{array}$ & $\begin{array}{l}10 \\
12\end{array}$ \\
\hline 7 & Arterial & $\begin{array}{l}\text { Preconexión } \\
\text { Post conexión temprana } \\
\text { Post conexión tardía }\end{array}$ & $\begin{array}{l}7,46 \\
7,46 \\
7,46\end{array}$ & $\begin{array}{l}27 \\
25 \\
25\end{array}$ & $\begin{array}{l}87 \\
89 \\
76\end{array}$ & $\begin{array}{l}19,3 \\
17,5 \\
17,9\end{array}$ & $\begin{array}{l}96 \\
97 \\
97\end{array}$ & $\begin{array}{l}10 \\
10 \\
10\end{array}$ \\
\hline 19 & Arterial & $\begin{array}{l}\text { Preconexión } \\
\text { Post conexión temprana }\end{array}$ & $\begin{array}{l}7,21 \\
7,35\end{array}$ & $\begin{array}{l}63,4 \\
51,1\end{array}$ & $\begin{array}{l}59 \\
54\end{array}$ & $\begin{array}{l}27,8 \\
28,6\end{array}$ & $\begin{array}{l}94 \\
98\end{array}$ & $\begin{array}{l}15 \\
15\end{array}$ \\
\hline
\end{tabular}

(*) Referido a descripción de los pacientes tabla 1.

duración de períodos de ventana, uso de válvulas de fonación y el tiempo total de uso de BiPAP durante el día. En la evolución de los pacientes, se registraron los siguientes eventos: mortalidad, desconexión o decanulación (se consignó el funcionamiento de alarmas, deterioro clínico o desaturación atribuible al episodio), eventos de hipoventilación (hipercapnea, $\mathrm{PaCO}_{2} \geq 45$ mmhg) o hiperventilación (hipocapnea, $\mathrm{PaCO}_{2}$ $<30 \mathrm{mmhg}$ ), colonización de la vía aérea (cultivos cuantitativos mensuales en pacientes hospitalizados en Hospital Josefina Martínez), número y duración de infecciones respiratorias, definiendo: a) Traqueobronquitis como aumento en las secreciones traqueobronquiales, que requirió aumentar el número de aspiraciones 
rutinarias de la vía aérea por más de 48 horas; b) Neumonía como episodio de fiebre mayor de 38,5 ${ }^{\circ} \mathrm{C}$ y síndrome de condensación; c) Atelectasia como episodio asociado a la disminución del murmullo vesicular en un área de auscultación de los campos pulmonares que requirió aumento de la kinesioterapia respiratoria (KTR) o aumento del diferencial de presión IPAP-EPAP.

\section{Resultados}

Veinte pacientes están recibiendo VMP con BiPAP a través de TQT. Siete pacientes se encuentran hospitalizados en el Hospital Josefina Martínez, 5 se encuentran institucionalizados en UCI/intermedio de su hospital de base de acuerdo a la región que pertenecen y 8 están en su domicilio. Estos últimos reciben los cuidados de enfermería y kinesiología en forma regular según las especificaciones del Programa de Asistencia Ventilatoria No Invasiva (www.avni. cl). Además cuentan con el equipamiento necesario para su apropiada monitorización cardiorrespiratoria.

La mediana de edad de los pacientes es de 3,5 años, con un rango de edad entre 3 meses y 17 años, según la etapa de ciclo vital la mayor parte de los pacientes corresponden a lactantes $\mathrm{y}$ preescolares, 11 pacientes son del género masculino.

Catorce pacientes tienen enfermedades neuromusculares con compromiso primario de la bomba respiratoria, siendo más frecuente los diagnósticos de Atrofia Espinal tipo I, Distrofia Muscular Congénita y Miopatía Congénita. Seis pacientes presentan daño pulmonar crónico y/o traqueomalacia/broncomalacia severa (tabla 3 ). La duración de la ventilación con BiPAP a través de TQT varía entre un mes y 5 años siendo la mediana 6,5 meses. Los rangos de presiones utilizados fueron: IPAP 8 a $20 \mathrm{cmH}_{2} \mathrm{O}$ y EPAP 4 a $8 \mathrm{cmH}_{2} \mathrm{O}$. Se utilizó modalidad asistida/ control (S/T) en 18 pacientes, la frecuencia de respaldo fue de 8 a 22 respiraciones por minuto. Seis pacientes requieren oxígenoterapia complementaria, con flujos menores a 2 1/min. Las características clínicas, parámetros ventilatorios, requerimientos de oxígeno y uso de válvulas de fonación se presenta en la tabla 3.
En siete pacientes hospitalizados en el Hospital Josefina Martínez se realizó medición de los flujos en el sistema una vez establecida la ventilación mecánica con BiPAP a través de TQT, la tabla 4 muestra la mediana, rangos y DS de estas mediciones. La mediana resultante de la diferencia de los flujos espiratorios antes y después del portal exhalatorio (leak) fue de $41 / \mathrm{min}$ con un rango de 2 a 11,5 1/min. El flujo generado por los BiPAP para mantener las presiones elegidas, flujo inspiratorio preportal, fue de 14,5 1/min con un rango de 7 a 29 1/min. La medición de los flujos inspiratorios después de este leak registraron una tasa de reducción de 5,5 1/min cuyo rango fue de 3 a 11 1/min (38\%). En estos pacientes, la presión trasmitida a la vía aérea y generada en el circuito de conexión se midió con un monitor de presión (Respironics, USA) inmediatamente antes de la TQT, observando para todos los registros concordancia con los valores planificados en el BiPAP. El uso del BiPAP en estos pacientes fue continuo, las 24 horas del día, en 12 niños, 5 pacientes realizan períodos de ventana y 3 sólo utilizan el BiPAP al dormir.

Se utilizó válvula de fonación en 8 pacientes que tuvieron ausencia de obstrucción de la vía aérea supraostoma, buena tolerancia clínica y en quienes la manometría de la presión subglótica al inicio de la espiración fue menor de $12 \mathrm{~cm}$ de $\mathrm{H}_{2} \mathrm{O}^{15,16}$. En 3 pacientes que no toleraban períodos de desconexión del BiPAP, se utilizó válvulas de Passy-Muir y en 5, que toleraban períodos de ventana durante su uso, válvulas Shiley. En 6 pacientes hospitalizados en el Hospital Josefina Martínez ventilados previamente con un ventilador mecánico convencional (IVent de Versamed o Vela de Vyasis) se registraron los gases sanguíneos antes y después de entregar VMP con BiPAP a través de TQT. En ellos se observó una tendencia a disminuir la $\mathrm{PaCO}_{2}$ (Pearson 0,68 $\mathrm{p}<0,05$ ). Tabla 5 .

\section{Complicaciones}

Durante el período reportado falleció un lactante de 16 meses con distrofia muscular congénita por una sepsis abdominal. No hubo mortalidad asociada al uso de ventilación no invasiva, 
neumotórax, traqueobronquitis ni atelectasias. En un paciente con BiPAP en modalidad S/T y opción AVAPS se pesquisó dentro de las primeras 48 horas de conexión hiperventilación sin complicaciones clínicas y que se solucionó disminuyendo el respaldo de frecuencia respiratoria durante la vigilia y ajustando el volumen minuto objetivo. Un paciente presentó hipoventilación, asociado a apneas centrales sin repercusión clínica, que logro manejarse con aumento de la FR de respaldo. Un paciente presentó un episodio de desconexión asociado a desaturación y bradicardia que ocurrió al movilizarlo durante el baño y que se resolvió sin incidentes, las alarmas en este caso funcionaron pero no fueron escuchadas por ruido ambiental del lugar, las alarmas del monitor permitieron detectar la condición descrita. Un paciente presentó un episodio de neumonía durante la VMP, en él se aisló un neumococo sensible en el cultivo cuantitativo de secreción traqueal, que respondió a las 48 horas de uso de ceftriaxona.

\section{Discusión}

Las novedades tecnológicas en ventilación mecánica no invasiva, principalmente como apoyo ventilatorio nocturno, han permitido tratar a un grupo creciente de adultos y niños con síndrome de apnea obstructiva (SAOS), hipoventilación nocturna secundaria a enfermedades neuromusculares y cifoescoliosis, hipoventilación central por compromiso anatómico o funcional del tronco cerebral y pacientes con fibrosis quística como soporte previo al trasplante pulmonar ${ }^{6,7-20}$. No obstante, en aquellos pacientes en los cuales los requerimientos de ventilación prolongada se deben extender más allá de la ventilación nocturna, es mejor tolerada y más segura la traqueostomía que el uso de interfases no invasivas. En esta situación, disminuyen los riesgos asociados a las mascarillas, tales como escaras, distensión gástrica, alteraciones del macizo facial y retención de $\mathrm{CO}_{2}{ }^{1}$.

Diferentes recomendaciones apuntan al beneficio de equipos de ventilación mecánica ciclados por tiempo y preferentemente limitados por presión para la entrega de ventilación domiciliaria. Es así, como una nueva genera- ción de equipos con turbina y con compresor permiten entregar flujo continuo, FR de respaldo y presión de soporte ventilatoria (PSV), optimizando modalidades de ventilación mandatoria intermitente sincronizada (SIMV) donde el esfuerzo del paciente permite un ciclo asistido al usar como umbral de disparo o trigger variaciones en el flujo o presión en la vía aérea de tal modo de minimizar el trabajo respiratorio por el paciente ${ }^{21}$.

El desempeño mostrado por estos equipos clásicos de ventilación mecánica tiene la ventaja de permitir el traslado seguro de los enfermos a sus domicilios, logrando reintegrar al paciente en el ámbito familiar, social, escolar y por lo tanto, mejorando la calidad de vida de los pacientes y su entorno familiar ${ }^{22}$. La elección de un BiPAP como modalidad de VMP a través de traqueostomía es posible del punto de vista fisiológico. Sin duda es más económico que el uso de un ventilador convencional, una vez resueltos aspectos de seguridad y desempeño, como son la ausencia de alarmas efectivas, el riesgo de hipoventilación, flujos elevados que limiten la tolerancia del enfermo o puedan generar hiperpresión y neumotórax.

La mayoría de nuestros pacientes previo a la conexión a BiPAP se encontraba en VMP con equipos convencionales en UCIP o en ventilación domiciliaria. La indicación de BiPAP a través de traqueostomía se fundamentó en los criterios de selección señalados, considerando la buena tolerancia a la PSV o a períodos de desconexión y en quienes el sistema de presión bi-nivelada venció apropiadamente la gradiente de presión determinada por la distensibilidad del pulmón y caja torácica, como por la resistencia en la vía aérea central y periférica.

En nuestra metodología de ventilación con un monocircuito se resolvió la necesidad de un portal exhalatorio creando una perforación de $2,5 \mathrm{~mm}$ en el conector interpuesto entre el corrugado y la traqueostomía, permitiendo no sólo impedir la recirculación del $\mathrm{CO}_{2}$, sino también mantener una tasa de flujo espiratorio suficiente para la activación de las alarmas de desconexión, apnea y volumen minuto bajo cuando se uso la modalidad AVAPS. Del mismo modo se logro una reducción de los flujos inspiratorios, que sin alterar las presiones plani- 
ficadas posiblemente ayudó a mejorar la tolerancia y comodidad del paciente.

Un estudio en adultos en fase de destete de la VMI comparó un ventilador convencional en modalidad CPAP $5 \mathrm{~cm}$ de $\mathrm{H}_{2} \mathrm{O}+5 \mathrm{~cm}$ de $\mathrm{H}_{2} \mathrm{O}$ de PSV con el funcionamiento para presiones equivalentes en un BiPAP ST/D, EPAP $5 \mathrm{~cm}$ e IPAP de $10 \mathrm{~cm}$ de $\mathrm{H}_{2} \mathrm{O}^{23}$. El generador de flujo fue equivalente al respirador convencional en minimizar el trabajo respiratorio, siendo igualmente eficaz y seguro en ventilar pacientes con demandas ventilatorias parciales que se recuperan de una insuficiencia respiratoria aguda, varios de ellos ventilados a través de una traqueostomía.

Otro estudio en adultos comparando un ventilador convencional (Evita IV) gatillado por flujo inspiratorio $(2 \mathrm{~L} / \mathrm{m})$ y por la disminución a 1/4 del Qmax para liberar la espiración, con un BiPAP (Visión) en que el algoritmo de disparo se determina por la forma de la curva de flujo (autotrack de Respironics) demostró que esta última opción es más sensible y eficiente en disminuir el trabajo inspiratorio que las modalidades clásicas de PSV como también logró una mejor sincronía en pacientes con insuficiencia respiratoria aguda ${ }^{24}$.

En nuestra experiencia, todos los pacientes pudieron ser apropiadamente ventilados con las modalidades ofertadas por el BiPAP, requiriendo un rango de presiones bajas o moderadas, lo cual muy probablemente incidió en la buena tolerancia a este tipo de VMP. Independiente de la edad, aquellos pacientes que toleraban en ventilación convencional estrategias de PSV se adaptaron con buena sincronía a la modalidad de BiPAP en asistida/control o asistida en aquellos con demandas ventilatorias parciales. Las tasas de flujo generadas durante la inspiración compatibilizaron el uso de válvulas de fonación (Passy-Muir) cuando las manometrías de la vía aérea lo permitían ${ }^{15,16}$.

Se podría especular que los beneficios de los equipos utilizados sobre la carga de trabajo de la musculatura respiratoria y sensibilidad de los flujos pudiesen incidir como un factor favorecedor del entrenamiento respiratorio. Sólo en una paciente escolar con síndrome de Escobar en que se pudo realizar entrenamiento con válvulas de umbral regulable IMT (ocluyendo la cánula) se pudo comprobar aumento significativo de la Pimax ${ }^{13}$. Nuevamente el rol del BiPAP en este logro es sólo especulativo.

A lo largo del tiempo, en aquellos pacientes con demandas parciales de soporte ventilatorio, se comprobó aumento en el tiempo total de desconexión, situación que claramente favorece la autonomía y las posibilidades de neurorehabilitación. Al no existir un grupo control es difícil especular si la VMI con BiPAP a través de traqueostomía juega algún rol en estos logros.

Conociendo las debilidades de este reporte descriptivo y sin un grupo control, parecen existir elementos clínicos que permiten sugerir que en este grupo particular de niños, en condición clínica estable, el uso de BiPAP a través de traqueostomía es una modalidad de VMP factible en suplir sus demandas ventilatorias.

\section{Conclusión}

El presente trabajo sugiere que el uso de BiPAP a través de traqueostomía en pacientes con criterios estrictos de selección es un método de VMP cuya implementación es factible. Se requiere un mayor número de estudios para lograr definir los costos, beneficios y riesgos potenciales de esta estrategia ventilatoria comparándola con los equipos tradicionales para entregar VMP en modalidad de ventilación mandatoria intermitente sincronizada. Se requieren definir índices de seguridad, eficiencia y eficacia específicos.

\section{Agradecimientos}

A todos los profesionales del Programa Chileno AVNI y del Hospital Josefina Martínez, por su labor incondicional con nuestros pacientes en pos de lograr su mejor manejo y calidad de vida.

\section{Referencias}

1.- Norregaard $O$ : Noninvasive Ventilation in Children. Eur Respir J. 2002; 20: 1332-42.

2.- Mellies U, Ragette R, Dohna C, Boehm H, Voit T, Teschler H: Long-term Noninvasive Ventilation in Children and Adolescents with Neuromuscular Disorders. Eur Respir J 2003; 22: 631-6. 
3.- Simonds A: Recent Advances in Respiratory Care for Neuromuscular Disease. Chest 2006; 130: 1879-86.

4.- Graham R, Fleegler E, Robinson W: Chronic Ventilator Need in the Community: A 2005 Pediatric Census of Massachusetts. Pediatrics 2007; 119: e12807.

5.- Simonds $A K$ : Respiratory support for the severely handicapped child with neuromuscular disease: ethics and practicality. Semin Respir Crit Care Med 2007; 28: $342-54$

6.- Young HK, Lowe A, Fitzgerald DA, et al: Outcome of noninvasive ventilation in children with neuromuscular disease. Neurology 2007; 68: 198-201.

7.- Ward S, Chatwin M, Heather S, Simonds A: Randomised controlled of non-invasive ventilation for nocturnal hypoventilation in neuromuscular and chest wall disease patients with daytime normocapnia. Thorax 2005; 60: 1019-24.

8.- Estopa Miró R, Villasante C, Lucas P, et al: Normativa sobre la ventilación mecánica en domicilio. Arch Bronconeumol 2001; 37: 142-9.

9.- Bertrand P, Fielhmann E, Lizama M, Holmgren N, Silva M, Sánchez I: Asistencia ventilatoria domiciliaria en niños chilenos: 12 años de experiencia. Arch Bronconeumol 2006; 42: 165-70.

10.- Make B, Hill N, Goldberg A, et al: Mechanical ventilation beyond the intensive care unit. Report of a consensus conference of the American College of Chest Physicians. Chest 1998; 113: 290-344.

11.- Ministerio de Salud: Programa Nacional de Asistencia Ventilatoria No Invasiva en Atención Primaria de Salud: Normas Técnicas. Revista Neumología Pediátrica 2007; 2: 38-48.

12.- Prado F, Salinas P, Astudillo P, Mancilla P, Méndez, M: Ventilación mecánica invasiva domiciliaria: Una propuesta para un nuevo Programa. Revista Neumología Pediátrica 2007; 2: 49-60.

13.- Torres $R$, Kuo $C$, Vera $R$, Espinoza $S$, Romero J: Entrenamiento muscular en paciente traqueostomizado: A propósito de un caso. Revista Neumología Pediátrica 2007; 2: 61-3.

14.- Ministerio de Salud, Subsecretaria de Redes Asistenciales: Resolución Exenta $N^{\circ} 372$ del 29 de febrero del 2008: Programa de Asistencia Ventilatoria No Invasiva en Atención Primaria de Salud y Protocolo de Ventila- ción Mecánica Invasiva en APS.

15.- Holmgren N, Brockmann P, Bertrand P: Medición de presión al final de espiración como factor predictor de tolerancia a válvula de fonación en pacientes traqueostomizados. V Curso Internacional y II Congreso de Neumología Pediátrica. Revista Neumología Pediátrica 2006; 1: 157.

16.- Hull EM, Dumas HM, Crowley RA, Kharasch VS: Tracheostomy speaking valves for children: tolerance and clinical benefits. Pediatr Rehabil 2005; 8: 214219.

17.- Gilgoff R, Gilgoff I: Long-term follow-up of home mechanical ventilation in young children with spinal cord injury and neuromuscular conditions. The Journal of Pediatrics 2003; 142: 476-80.

18.- Panitch $H$ : Respiratory Issues in the Management of Children with Neuromuscular Disease. Respir Care 2006; 51: 885-893.

19.- Efrati O, Kremer MR, Barak A, et al: Improved survival following lung transplantation with long-term use of bilevel positive pressure ventilation in cystic fibrosis. Lung 2007; 185: 73-9.

20.- Fauroux B, Boffa C, Desguerre I, Estournet B, Trang H: Long-term noninvasive mechanical ventilation for children at home: a national survey. Pediatr Pulmonol 2003; 35: 119-25.

21.- Battisti A, Tassaux D, Janssens JP, Michotte JB, Jaber S, Jolliet P: Performance characteristics of 10 home mechanical ventilators in pressure-support mode: a comparative bench study. Chest 2005; 127: 178492.

22.- Earle RJ, Rennick JE, Carnevale FA, Davis GM: "It's okay, it helps me to breathe": the experience of home ventilation from a child's perspective. J Child Health Care 2006; 10: 270-82.

23.- Patel RG, Petrini MF: Respiratory muscle performance, pulmonary mechanics, and gas exchange between the bipap S/T-D system and the Servo Ventilator 900C with bilevel positive airway pressure ventilation following gradual pressure support weaning. Chest 1998; 114: 1390-6.

24.- Prinianakis G, Kondili E, Georgopoulos D: Effects of the flow waveform method of triggering and cycling on patient-ventilator interaction during pressure support. Intensive Care Med 2003; 29: 1950-9. 